\title{
Development and evaluation of floating microspheres of curcumin in alloxan-induced diabetic rats
}

\author{
Kapil Kumar ${ }^{1 *}$, Navin Chandra Pant ${ }^{1}$, S Ahmad ${ }^{1}$, MV Fateh ${ }^{1}$, AK Rai ${ }^{2}$, Bipin \\ Verma $^{3}$ and Himanshu Chaurasia ${ }^{4}$ \\ ${ }^{1}$ Global Institute of Pharmaceutical Education and Research, Kashipur, U.K., ${ }^{2}$ Pranveer Singh Institute of Technology, Kanpur, \\ U.P., ${ }^{3}$ Government Polytechnic, Kashipur, U.K. ${ }^{4}$ AVIP, Saharanpur, Gangoh, U.P. India
}

*For correspondence: Email: kapil5november@gmail.com; Tel: +91-99977-22274

\begin{abstract}
Purpose: To prepare and evaluate floating microspheres of curcumin for prolonged gastric residence and to study their effect on alloxan-induced diabetic rats.

Methods: Floating microsphere were prepared by emulsion-solvent diffusion method, using hydroxylpropyl methylcellulose, chitosan and Eudragit $S 100$ polymer in varying proportions. Ethanol/dichloromethane blend was used as solvent in a ratio of 1:1. The floating microspheres were evaluated for flow properties, particle size, incorporation efficiency, as well as in-vitro floatability and drug release. The anti-diabetic activity of the floating microspheres of batch FM4 was performed on alloxaninduced diabetic rats.

Result: The floating microspheres had particle size, buoyancy, drug entrapment efficiency and yield in the ranges of 255.32 - $365.65 \mu \mathrm{m}, 75.58$ - 89.59, 72.6 - 83.5, and $60.46-80.02 \%$, respectively. Maximum drug release after $24 \mathrm{~h}$ was $82.62 \%$ for formulation FM4 and 73.879, 58.613 and $46.106 \%$ for formulations FM1, FM2, and FM3 respectively. In-vivo data obtained over a 120-h period indicate that curcumin floating microspheres from batch FM4 showed the better glycemic control than control and a commercial brand of the drug.

Conclusion: The developed floating curcumin delivery system seems economical and effective in diabetes management in rats, and enhances the bioavailability of the drug.
\end{abstract}

Keywords: Gastro-retentive, Sustained release, Bioavailability, Curcumin, Floating microspheres, Diabetes

Tropical Journal of Pharmaceutical Research is indexed by Science Citation Index (SciSearch), Scopus, International Pharmaceutical Abstract, Chemical Abstracts, Embase, Index Copernicus, EBSCO, African Index Medicus, JournalSeek, Journal Citation Reports/Science Edition, Directory of Open Access Journals (DOAJ), African Journal Online, Bioline International, Open-J-Gate and Pharmacy Abstracts

\section{INTRODUCTION}

Glucose is necessary for health because it's an important source of energy for the cells that make muscles and tissues. When the blood glucose increases for example, after eating food material, insulin is released from the pancreas to normalize the glucose level [1]. Diabetes mellitus is a condition in which a person has a high blood sugar level, either because the body doesn't produce enough insulin, or because body cells don't properly respond to the insulin that is produced. If the body cells do not absorb glucose, the glucose accumulates in the blood, leading to vascular, neuron, and other complications.

Diabetes mellitus is the commonest endocrine disorder that affects more than 171 million people worldwide and according to a recent study, this population may be increased 366 million by 2030 [2]. 
Natural products have received considerable attention for the management of diabetes and its complications which have reached epidemic proportion worldwide. The spice turmeric, which is derived from the root of the plant Curcuma longa, has been described as a treatment for diabetes in Ayurvedic and traditional Chinese traditional medicine for thousands of years [3].

Curcumin, a powerful antioxidant, is a component of turmeric found in the Curcuma longa plant and has been used for centuries in treating inflammatory ailments. Its antiinflammatory properties have been attributed to the main components of turmeric, the curcuminoids including Curcumin. Curcumin is a potent scavenger of reactive oxygen and nitrogen species such as hydroxyl radicals and nitrogen dioxide radicals [4]. The possible mechanisms of the effect of curcumin on glycemia in diabetes models may be explained as follows. First, curcumin could attenuate tumor necrosis factor- (TNF- $\alpha$ ) levels and plasma free fatty acids. It also inhibits nuclear factor-kappa $\beta$ (NF- $\beta$ ) activation and protein carbonyl [5] lipid peroxidation. Furthermore curcumin is involved in activating of enzymes in liver, which are associated with glycolysis, gluconeogenic, and lipid metabolic process [6].

Floating microspheres are gastroretentive drug delivery systems based on a non-effervescent approach. These microspheres are characteristically free-flowing powders having a size < $199 \mu \mathrm{m}$ and remain buoyant over gastric contents for a prolonged period. As the system floats over gastric contents, the drug is released slowly at the desired rate, resulting in increased gastric retention with reduced fluctuations in plasma drug concentration [7]. The objective of present study was to develop a floating drug delivery system of Curcumin and to study their therapeutic effectiveness on alloxan induced diabetic rats.

\section{EXPERIMENTAL}

Curcumin was a gift from Krish Enteprizes, Mumbai, India. HPMC and Eudragit S 100 were received as a gift samples from Glukem Pharmaceuticals (P) Ltd, India. All other chemicals used were of analytical grade.

\section{Preparation of floating microspheres}

The floating microspheres were prepared by emulsion solvent diffusion method [8]. The drug and polymer ratio used are shown in Table 1. The materials were weighed and added to a mixture of dichloromethane and ethanol. The slurry was introduced into $250 \mathrm{ml}$ beaker containing tween 80 while being stirred at 750 rpm by mechanical stirrer (Eltek Motor, Mumbai) for $1 \mathrm{~h}$ at room temperature. The floating microspheres were collected by decantation while the non-floating microspheres were discarded along with polymer residues and washed thrice with n-hexane. The collected microspheres were dried overnight in an oven at $40 \pm 2{ }^{\circ} \mathrm{C}$ and stored in a desiccators containing calcium chloride as a desiccant.

Table 1: Composition of formulations

\begin{tabular}{lcccc}
\hline Ingredient & FM1 & FM2 & FM3 & FM4 \\
\hline Curcumin $(\mathrm{mg})$ & 100 & 100 & 100 & 100 \\
Eudragit S100 $(\mathrm{mg})$ & 150 & 250 & 500 & - \\
Chitosan $(\mathrm{mg})$ & - & - & 150 & 250 \\
HPMC $(\mathrm{mg})$ & - & - & 150 & 200 \\
Dichloromethane:ethanol & $5: 5$ & $5: 5$ & $5: 5$ & $5: 5$ \\
1:1 $(\mathrm{ml})$ & & & & \\
Tween $80(\mathrm{ml})$ & 2 & 2 & 2 & 2 \\
\hline
\end{tabular}

\section{Evaluation of floating microsphere}

\section{Particle size analysis}

The particle size was measured by microscopic technique. In this method suspension of floating microspheres was prepared using castor oil. A drop of suspension was mounted on a slide and observed under optical microscope (VN-7, Cosmo Lab, India) about 600 particles were measured with the help of the eye piece micrometer. All the microspheres in a field were counted [9].

\section{Measurement of bulk density}

In this method, floating microspheres were transferred to a measuring cylinder and tapped manually till a constant volume was obtained. This volume is bulk volume and it included the true volume of the powder and the void space among the microspheres. Bulk density was computed as the ratio of the mass of the microsphere to the bulk volume [8].

Bulk Density $=$ (Mass of microspheres)/(Bulk Volume)Measurement of tapped density

In this method floating microspheres were transferred to a measuring cylinder and tapped for 100 times. After tapping volume of microspheres was visually examined. The ratio of mass of microspheres to volume of microspheres after tapping gave the tapped density of the floating microspheres [10]. 
Tapped Density $=$ (Mass of microspheres)/(Volume of microspheres after tapping)

\section{Measurement of Carr's (compressibility) index}

This parameter was calculated from bulk density (the ratio of weighed quantity of microspheres to its volume), DP, and tapped density as in Eq 1.

Compressibility index $=($ DT-DP $) / D X \times 100 \ldots(1)$

\section{Measurement of Hausners ratio}

Hausners ratio of microspheres was determined as the ratio of tapped density to bulk.

Hausners ration $=($ Bulk density $) /($ Tapped density)

Values $<1.25$ indicate good flow (= $20 \%$ Carr), while values $>1.25$ indicate poor flow [8].

\section{Evaluation of angle of repose}

Angle of repose $(\theta)$ of the microspheres, which measures the resistance to particle flow, was determined by a fixed funnel method4. The height $(h)$ and radius $(r)$ of the powder cone was measured and angle of repose was calculated using Eq 2 [10].

$\theta=\tan ^{-1} \mathrm{~h} / \mathrm{r}$

\section{Measurement of drug entrapment efficiency}

Microspheres equivalent to $50 \mathrm{mg}$ of the drug were taken for evaluation. The amount of drug entrapped was estimated by crushing the microspheres and extracting with aliquots of 0.1 $\mathrm{N} \mathrm{HCl}$ repeatedly. The extract was transferred to a $100 \mathrm{ml}$ volumetric flask and the volume was made up using $0.1 \mathrm{~N} \mathrm{HCl}$. The solution was filtered and the absorbance was measured at $254 \mathrm{~nm}$ by UV spectrophotometer (Shimadzu) against appropriate blank [10]. The amount of drug entrapped in the microspheres was calculated as the ratio of the actual amount of drug found in the microsphere to the theoretical drug load, expressed as a percentage.

\section{Determination of microsphere yield}

The prepared microspheres were weighed. The measured weight was divided by the total amount of all non-volatile components which were used for the preparation of the microspheres, and then expressed as a percentage.

\section{Assessment in-vitro buoyancy}

Microspheres (300 mg) were spread over the surface of a USP XXIV dissolution apparatus type II (Electro Labs, Mumbai) filled with $900 \mathrm{ml}$ of $0.1 \mathrm{~N}$ hydrochloric acid containing $0.02 \%$ tween 80. The medium was agitated with a paddle rotating at $100 \mathrm{rpm}$. The floating and the settled portions of microspheres were recovered separately. The microspheres were dried and weighed. Buoyancy percentage was calculated as the ratio of the mass of the microspheres that remained floating to the total mass of the microspheres, expressed as a percentage [11].

\section{In-vitro drug release studies}

A USP basket apparatus was used to study invitro drug release from microspheres. In the present study drug release was studied using a modified USP XXIV dissolution apparatus type I ((Electro Labs, Mumbai)) at $100 \mathrm{rpm}$ in distilled water and $0.1 \mathrm{~mol} \mathrm{~L}^{-1} \mathrm{HCL}(\mathrm{pH} 1.2)$ as dissolution fluids $(900 \mathrm{ml})$ maintained at $37 \pm 1$ ${ }^{\circ} \mathrm{C}$. Aliquots were withdrawn at periodical intervals and analyzed spectrophotometrically at $254 \mathrm{~nm}$. The volume was replenished with the same amount of fresh medium to maintain the sink condition. All experiments were performed in triplicate [12].

\section{Assessment of hypoglycaemic activity}

The approval of the Institutional Animal Ethics Committee was obtained before starting the study. The study was carried out as per the guidelines compiled by the Committee for the Purpose of Control and Supervision of Experiments on Animal (CPCSEA), Ministry of Culture, Government of India and all the study protocols were approved by the local institutional Animal Ethics Committee PSIT, Kanpur, India (R.N.-1237/AC/CPCSEA). Also an international protocol for conducting experiments on animals were followed [13]. Healthy wistar rats of either sex having weight 150 - $200 \mathrm{~g}$ were selected for this activity. They were housed in standard condition of temperature $\left(25 \pm 2{ }^{\circ} \mathrm{C}\right)$ with $12 \mathrm{~h}$ light per day cycle. Before induction of diabetes weigh and normal glucose levels of rats were determined and recorded as Day 0 . The acclimatized animals were fasted for $24 \mathrm{~h}$ with water ad libitum and injected intraperitoneally freshly prepared alloxan monohydrate in normal at a dose of $150 \mathrm{mg} / \mathrm{kg}$ body weight [14]. All animals were returned to their cages and given free access to food and water. Blood glucose 
levels were monitored by using a Glucometer (Pulsatum Health Care Pvt. Ltd., India) after $72 \mathrm{~h}$ of injection and recorded as 1 st day. Only rats with fasting blood glucose level greater than 250 $\mathrm{mg} / \mathrm{ml}$ were considered diabetic and included in this study. Diabetic rats were randomly assigned to three groups, each group contains six animals.

The animals were grouped as follows:

a) Diabetic control: normal saline solution

b) Floating microspheres formulation of Group FM4: $(200 \mathrm{mg} / \mathrm{kg})$

d) Standard group: Glibenclamide: $(10 \mathrm{mg} / \mathrm{kg}$ body weight orally by dissolving in normal saline solution).

Glucometer (Pulsatum Health Care Pvt Ltd, India) was used to monitor blood glucose levels of the rats. Blood samples were obtained from the cut tail tip of the conscious rat and glucose test strip soaked with blood and then inserted to be read by the glucometer. Blood glucose levels were examined after $2,12,24,72,120 \mathrm{~h}$ of orally administration of test drugs.

\section{Statistical analysis}

To ascertain drug release mechanism and release rate, the release data were model fitted using PCP Disso V3.0 dissolution software. Experimental results were expressed as mean \pm SD. Student's t-test and one-way analysis of variance (ANOVA) were applied to check significant differences in drug release from different formulations. Differences were considered to be statistically significant at $p<$ 0.05 .

\section{RESULTS}

\section{Properties of floating microspheres}

The properties of the microspheres are shown in Table 2, Table 3, Table 3 and Fig 1. Mean particle size significantly increased with increasing polymer concentration this was due to high viscosity of polymer concentration require high energy for breaking of droplets. The microspheres were more difficult to disperse; these results in larger size of microspheres. The drug entrapment efficiency of different batches of floating microspheres was found in range of 73.56 - 80.02. A large proportion of the microspheres remained floating after $12 \mathrm{~h}$. Floatation might have been influenced by the low bulk and tapped densities of the microspheres. Buoyancy for all the batches was above $75 \%$, which was studied for $12 \mathrm{~h}$. Mean buoyancy ranged from 75.58 to $89.59 \%$. Highest buoyancy was obtained with formulation FM4. In general with increase in the amount of polymer (chitosan), there was an increase in the buoyancy percentage.

The release profiles of curcumin varied with the different formulations. Curcumin release from these microspheres was slow, extended and dependent on the type of polymer used. In-vitro release study shows maximum release for formulation FM4, (82.62 \%), during the studies of $24 \mathrm{~h}$.

Table 2: Characteristics of floating microspheres

\begin{tabular}{lcccc}
\hline Parameter & FM1 & FM2 & FM3 & FM4 \\
\hline Particle size $(\mu \mathrm{m})$ & $255.32 \pm 0.135$ & $263.87 \pm 0.253$ & $321.52 \pm 0.332$ & $365.65 \pm 0.158$ \\
Bulk density $\left(\mathrm{g} / \mathrm{cm}^{3}\right)$ & $0.154 \pm 0.014$ & $0.160 \pm 0.024$ & $0.163 \pm 0.015$ & $0.170 \pm 0.039$ \\
Tapped density $\left(\mathrm{g} / \mathrm{cm}^{3}\right)$ & $0.172 \pm 0.153$ & $0.212 \pm 0.230$ & $0.209 \pm 0.036$ & $0.218 \pm 0.126$ \\
Hausner,s ratio & $1.118 \pm 0.028$ & $1.193 \pm 0.005$ & $1.139 \pm 0.007$ & $1.146 \pm 0.142$ \\
Carr's index & $17.14 \pm 0.142$ & $20.53 \pm 0.234$ & $19.43 \pm 0.104$ & $15.0 \pm 0.208$ \\
Angle of repose & $17.510 \pm 0.016$ & $18.510 \pm 0.021$ & $20.870 \pm 0.113$ & $22.080 \pm 0.327$ \\
Buoyancy $(\%)$ & $75.58 \pm 0.134$ & $87.26 \pm 0.316$ & $84.61 \pm 0.138$ & $89.59 \pm 0.226$ \\
Yield $(\%)$ & $60.46 \pm 0.138$ & $61.12 \pm 0.118$ & $73.56 \pm 0.074$ & $80.02 \pm 0.062$ \\
Drug entrapment & $73.56 \pm 0.024$ & $79.25 \pm 0.047$ & $81.21 \pm 0.072$ & $82.45 \pm 0.035$ \\
Efficiency $(\%)$ & & & & \\
\hline
\end{tabular}




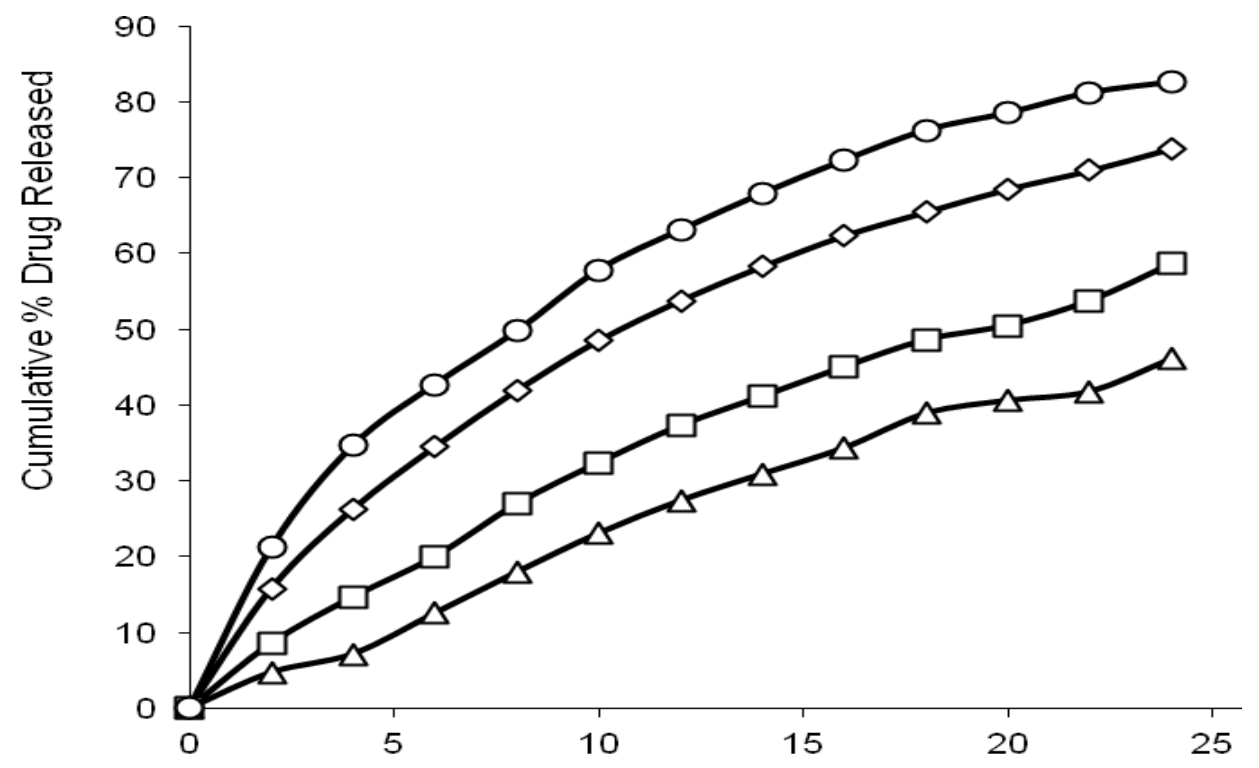

Time (Hrs)

Figure 1: Drug release profiles of curcumin from floating microspheres ( $\square \square \square \square \square \diamond=F M 1 ; \square \mathrm{FM} 2 ; \Delta=\mathrm{FM} 3 ; \mathrm{O}=$ FM4)

Table 3: In-vitro drug release kinetics of the formulations

\begin{tabular}{lccccccc}
\hline $\begin{array}{l}\text { Formulation } \\
\text { code }\end{array}$ & \multicolumn{2}{c}{ Zero order } & \multicolumn{2}{c}{ Higuchi model } & \multicolumn{2}{c}{ Korsemeyer-Peppas model } \\
& \multicolumn{2}{c}{} & & & & & \\
& $\mathbf{R}$ & $\mathbf{K}$ & $\mathbf{R}$ & $\mathbf{K}$ & Slope (n) & $\mathbf{R}$ & $\mathbf{K}$ \\
\hline FM1 & 0.8541 & 1.1454 & 0.9627 & 10.0362 & 0.6841 & 0.9641 & 5.1338 \\
FM2 & 0.8224 & 1.2632 & 0.9543 & 9.5253 & 0.6495 & 0.9678 & 5.0788 \\
FM3 & 0.9052 & 1.1566 & 0.9848 & 10.1451 & 0.6581 & 0.9732 & 4.0586 \\
FM4 & 0.9121 & 1.2882 & 0.9857 & 10.1244 & 0.6746 & 0.9664 & 6.1685 \\
\hline
\end{tabular}

Table 4: Anti-diabetic activity of floating microspheres of curcumin against alloxan-induced diabetic rats

\begin{tabular}{lcccccc}
\hline & \multicolumn{5}{c}{ Time (h) } \\
\cline { 2 - 7 } Group & $\mathbf{0}$ & $\mathbf{2}$ & $\mathbf{1 2}$ & $\mathbf{2 4}$ & $\mathbf{7 2}$ & $\mathbf{1 2 0}$ \\
\hline Diabetic Control & 362.42 & 364.49 & 372.61 & 380.82 & 390.83 & 400.28 \\
Floating microspheres, batch FM4 & 350.27 & 338.62 & 324.71 & 312.42 & 290.38 & 270.62 \\
Diabetic + Glibenclamide & 348.42 & 322.53 & 306.41 & 284.31 & 268.83 & 260.34 \\
\hline
\end{tabular}

$\mathrm{N}=6, p<0.05$

\section{DISCUSSION}

Drug absorption in the gastrointestinal tract is a highly variable process. Floating microspheres are promises to be a potential approach for gastric retention to enhance the bioavailability.

Floating microspheres as gastroretentive dosage forms precisely control the release rate of target drug to a specific site and facilitate an enormous impact on health care. After oral administration, these are retained in the stomach and release the drug there in a controlled and prolonged manner to allow the drug to be supplied continuously to its absorption sites in the upper gastrointestinal tract.
The present study is an attempt to formulate curcumin floating microspheres formulations with a view of improving its oral bioavailability and giving a prolonged release of drug, and also to study their effect on alloxan-induced diabetic rats.

Drug entrapment efficiency increased with increasing polymer concentration in floating microspheres. The high entrapment efficiency of curcumin is believed to be due to its poor aqueous solubility in disperse phase. The percentage entrapment efficiency increased with increase in polymer concentration because higher viscosity of chitosan solution reduces the diffusion of the drug in the surroundings which does not allow entrapped particle to escape easily. 
Eudragit $S 100$ is insoluble in acidic medium and also exhibits low permeability. It is an anionic copolymer of methacrylic acid and methyl methacrylate containing free carboxylic and ester groups [15]. The initial drug release significantly increased with an increase in the ratio of drug/polymer. An initial high release was observed due to the dissolution of surface adhered drug, whereas the latter release was due to the diffusion process, which is much slower when, compared to the initial release. The regression coefficient values for all these models are shown in Table 4. The models selected were zero order, Higuchi, Korsemeyer-Peppas. The zero order plots showed the zero order release characteristics of the formulation, which was confirmed by the correlation value which found to be nearer to one. Correlation value of Higuchi's plot revealed that the mechanism of drug release is diffusion. The in vitro kinetic data subjected to log time log drug release transformation $n$ plot (Peppas' model) revealed the fact that the drug release follows a super case II transport diffusion. In all the cases the best fit model was found to be Peppas with ' $n$ ' value between 0.6495 and 0.6841 , suggesting a non-Fickian (anomalous) release mechanism for the drug, i.e., erosion followed by diffusion controlled. Alloxan administration resulted in significant elevation of glucose level and reduction in body weight. The in-vivo study of curcumin floating microspheres (FM4) showed optimal glycemic control is best when compared with control and Marketed product.

Alloxan causes diabetes through its ability to destroy the insulin-producing beta cells of the pancreas. In vitro studies have shown that alloxan is selectively toxic to pancreatic beta cells, leading to the induction of cell necrosis. The cytotoxic action of alloxan is mediated by reactive oxygen species, with a simultaneous massive increase in cytosolic calcium concentration, leading to a rapid destruction of beta cells [16]. In light of the results, study indicates that floating microspheres of curcumin have good anti-diabetic activity.

\section{CONCLUSION}

The findings suggest that gastro retentive floating microspheres have the potentials to be developed as an efficient delivery system for enhanced bioavailability and controlled delivery of curcumin for diabetes therapy as it contains various complex chemical substance of different composition in the form of secondary plant metabolites in one or more parts of these plants.

\section{DECLARATIONS}

\section{Acknowledgement}

The authors are thankful to Dr AK Saxena ((ExChief Scientist and former Head, Division of Medicinal Chemistry, CDRI, Lucknow, India for their technical assistance and motivation during the work.

\section{Conflict of Interest}

No conflict of interest associated with this work.

\section{Contribution of Authors}

The authors declare that this work was done by the authors named in this article and all liabilities pertaining to claims relating to the content of this article will be borne by them.

\section{REFERENCES}

1. Ranjan $C$, Ramanujam $R$. Diabetes and insulin resistance associated disorders - Disease and the therapy. Curr Sci 2002; (83): 1533-1538.

2. Rother KI. Diabetes treatment-bridging the divide. New Engl J Med 2007; (356) 15: 1499-501.

3. Agarwal BB, Sundaram C, Malani N. Curcumin-the Indian solid gold. Advances in Experimental Medicine and Biology 2007; (595): 1-75.

4. Ruby AJ, Kuttan G, Dinesh BK, Rajasekharan KN, Kuttan R. Antitumor and antioxidant activity of natural curcuminoids. Cancer Lett 1995; 94: 79-83.

5. Suryanarayana $P$, Satyanarayana $A$, Balakrishna $N$. Effect of turmeric and curcumin on oxidative stress and antioxidant enzymes in streptozotocin-induced diabetic rat. Medical Science Monitor 2007; 13(12): 286-292.

6. Seo KI, Choi MS, Jung UJ. Effect of curcumin supplementation on blood glucose, plasma insulin, and glucose homeostasis related enzyme activities in diabetic mice. Molecular Nutrition and Food Research 2008; 52(9): 995-1004.

7. Chien YW. Novel drug delivery system. Marcel Dekker Inc Publications, New York 1992; 2(50): 16172.

8. Kawashima $Y$, Niwa $T$, Takeuchi $H$, Hino $T$, Itoh $Y$. Hollow microspheres for use as a floating controlled drug delivery system in the stomach. Pharm Sci 1992; 81: 135-140.

9. Parasuram RR, Moidutty L, Chetan H. Preparation and evaluation of delayed release aceclofenac microspheres. Asian J Pharm 2008; 2(4): 52-54.

10. Aulton ME. Pharmaceutics - The Science of Dosage Form Design, Churchill Livingstone, Elsevier Science Ltd; 2002; (2): pp 315-320.

11. Srivastava A. Floating microspheres of cimetidine formulation, characterization and in vitro evaluation. Acta Pharm 2005; 55: 277-285.

Trop J Pharm Res, September 2016; 15(9): 1824 
12. Maheswari U, Jain S, Bhadra D, Jain NK. Floating microspheres bearing acetohydroxamic acid for the treatment of $\mathrm{H}$. pylori. J Pharm Pharmacol 2003; 55: 1607-1613.

13. Kuhara T. Consensus recommendations on effective institutional animal care and use committees. Information on Overseas Technologies in Laboratory Animal Science 1988; 7: 14-17

14. Ko GJ, Kang YS, Han SY. Pioglitazone attenuates diabetic nephropathy through an anti-inflammatory mechanism in type 2 diabetic rats. Nephrol Dial Transplant 2008; 23 (9): 2750-60.
15. Korsmeyer RW, Gurny R, Doelker EM, Buri P, Peppas NA. Mechanism of solute release from porous hydrophilic polymers. Int J Pharm 1983; 15: 25-35.

16. Jorns A, Munday R, Tiedge M, Lenzen S. Comparative toxicity of alloxan, $\mathrm{N}$-alkyl-alloxans and ninhydrin to isolated pancreatic islets in vitro. J Endocrinol 1997; 155: 283-293.

17. Sharma $M$, Kohlib $S$, Dindac $A$. In-vitro and in-vivo evaluation of repaglinide loaded floating microspheres prepared from different viscosity grades of HPMC polymer. Saudi Pharm J 2015; 23(6): 675-682. 